EESTI NSV TEADUSTE AKADEEMIA TOIMETISED. XII KÖIDE

FOOSIKA-MATEMAATIKA- JA TEHNIKATEADUSTE SEERIA. 1963, NR. 3

ИЗВЕСТИЯ АКАДЕМИИ НАУК ЭСТОНСКОИ ССР. ТОМ ХІІ

СЕРИЯ ФИЗИКО-МАТЕМАТИЧЕСКИХ И ТЕХНИЧЕСКИХ НАУК. 1963, № 3

\title{
К ТЕОРИИ КОМБИНАЦИОННОГО РАССЕЯНИЯ СВЕТА МОЛЕКУЛОИ
}

\author{
К. РЕБАНЕ, \\ член-корреспондент Академни наук Эстонской ССР
}

T. РЕБАНЕ,

кандидат физико-математических наук

Дан новый метод расчета ннтенсивностей линий комбинационного рассеяния света. С помощью этого метода выполнено точное суммирование по промежуточным состояниям в формуле второго порядка теории возмущений для модели гармонического осциллятора. Рассмотрены некоторые общие вопросы второго порядка теорни возмущений.

\section{1. Введение}

В связи с начавшимся в последние годы детальным экспериментальным изучением интенсивностей комбинационного рассеяния света на молекулах $[1-3]$ повысился интерес к разработке уточненных математических методов теории этого явления (обзор современного состояния вопроса дан, например, Л. Л. Крушинским $\left[{ }^{4}\right]$; там же подробная библиография). Однако до самого последнего времени отсутствовали работы, в которых была бы удовлетворительно преодолена основная математическая трудность расчета интенсивностей линий комбинационного рассеяния, связанная с суммированием по промежуточным состояниям в соответствуюших формулах второго порядка теории возмущений. Совсем недавно Преэму $\left[{ }^{5,6}{ }^{6}\right.$ удалось с помощью разработанного им метода производящей функции точно выполнить такое суммирование и полуqить в виде гипергеометрических рядов формулы для интенсивностей линий комбинационного рассеяния света на колебаниях молекулы: При этом использовалось адиабатическое приближение и обычная модель колеблющейся молекулы - гармонический осциллятор, положение равновесия и частота которого изменяются в результате изменения электронного состояния.

В настоящей работе дается новый метод расчета интенсивности комбинационного рассеяния на основе точного суммирования формулы второго порядка теории возму. щений для модели гармонического осциллятора.

Задача комбинационного рассеяния является одной из типичных задач второго порядка теории возмущений, к которым приводят многие проблемы квантовой механики атомов, молекул и кристаллов. Весьма часто употребляемый способ решения лодобных задач - выделение одного или нескольких членов из бесконечного ряда никогда не является вполне удовлетворнтельным, а иногда ведет просто к неправильным выводам. Исследование и усовершенствование метөдов решения этих задач остается актуальным. Поэтому параграфы 5 и 9 посвящены некоторым общим соотношениям, которые, как нам представляется, полезно иметь в виду при решении задач высших порядков как нестационарной, так и стационарной теории возмушений. 


\section{2. Постановка задачи}

Интенсивность комбинационного рассеяния света $I_{m n}$ частоты $\omega+\omega_{m n}$ дается формулой (см., напр., у Д. И. Блохинцева [7], стр. 311):

$$
I_{m n}=\frac{\left(\omega_{m n}+\omega\right)^{4}}{3 c^{3}}\left|\frac{1}{h} \sum_{k}\left(\frac{\left(\vec{E} \vec{D}_{k n}\right) \vec{D}_{m k}}{\omega_{n k}-\omega}+\frac{\left.\overrightarrow{(E} \vec{D}_{m k}\right) \vec{D}_{k n}}{\omega_{m k}+\omega}\right)\right|^{2}
$$

Здесь $\vec{D}_{m n}$ - матричный элемент электрического дипольного перехода системы между состояниями $m$ и $n, \omega$ и $\vec{E}$ - частота и амплитуда электрического вектора падающей световой волны; изменение ее частоты $\omega_{m n}=\left(E_{m}-E_{n}\right) / h$.

Будем рассматривать в качестве рассеивающей системы двухатомную молекулу и исходить из адиабатического приближения БорнаОппенгеймера. Учтем вклад лишь одного возбужденного электронного состояния и примем, что в результате электронного перехода изменяется только положение равновесия колебания молекулы, частота же остается неизменной.

Задача нахождения $I_{m n}$ сводится в рассматриваемом случае, по существу, к вычислению сумм следуюшего типа:

$$
S_{i f}(\mu, a)=\sum_{k=0}^{\infty} \frac{P_{k f} P_{i k}}{\varepsilon_{k}-\mu},
$$

где

$$
\begin{gathered}
P_{k f}=\int \psi_{k} \widehat{P}_{\varphi_{i}} d x ; \\
\widehat{H}_{\mathrm{II}} \psi_{k}=\varepsilon_{k} \psi_{k} ; \quad \widehat{H}_{\mathrm{I}} \varphi_{f}=\varepsilon_{f} \varphi_{i} ; \quad \widehat{H}_{\mathrm{I}} \varphi_{i}=\varepsilon_{i} \varphi_{i} .
\end{gathered}
$$

Здесь введены следующие обозначения. $\widehat{P}$ - оператор возмущения, в качестве которого в силу адиабатического приближения выступаег матричный элемент электронного дипольного перехода; $\widehat{H}_{\mathrm{I}}$ и $\widehat{H}_{\mathrm{II}}-$ гамильтонианы гармонических осцилляторов, характеризующиеся одинаковой частотой $\omega_{0}$, но отличающиеся положениями равновесия, описывающие колебания ядер соответственно в исходном и промежуточном

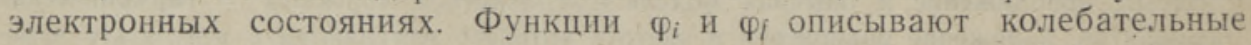
состояния исходного электронного состояния, между которыми совершается переход в акте комбинационного рассеяния. Поскольку мы не интересуемся здесь магнитными явлениями, то без ограничения общно сти можно считать, что рассматриваемые нами волновые функции вещественны. Параметр $a$ характеризует сдвиг положения равновесия осциллятора в результате электронного перехода.

Далее, ограничимся так называемым приближением Кондона, т. е. пренебрежем зависимостью электронного матричного элемента от колебательной координаты молекулы и будем рассматривать относительные интенсивности линий. Әтому соответствует $\widehat{P}=\mathrm{const}=1$.

Сделанные нами упрощающие предположения являются общепринятыми в современной теории молекул и во многих случаях приводят к хорошему согласию с результатами опыта. Уточнения теории, например 
учет отклонения от приближения Кондона и ангармоничности колебаний, дают, как правило, небольшие поправки к основному результату *. Поэтому можно считать, что после всех перечисленных приближений мы пришли к основной задаче комбинационного рассеяния света, для решения которой полностью сохранило актуальность точное выполнение суммирования в формуле (2).

\section{3. Обычная форма теории возмущений}

Рассмотрим переход гармонического осциллятора, обладающего частотой $\omega_{0}$, с уровня $i=0$ на уровень $f=1$. Этому случаю соответствует уменьшение частоты падающего света на величину $\omega_{0}$. В формуле (2) мы имеем конкретно:

$$
\begin{gathered}
\varepsilon_{k}=\frac{2 E_{k}}{h \omega_{0}}=2 k+1 \quad(k=0,1,2, \ldots) \\
P_{i k}=\int \varphi_{i} \psi_{k} d x ; \quad P_{k i}=\int \psi_{k} \varphi_{f} d x ; \\
\varphi_{i}=\varphi_{0}=\pi^{-\frac{1}{4}} e^{-\frac{(x-a)^{2}}{2}} ; \quad \varphi_{f}=\varphi_{1}=\sqrt{2} \pi^{-\frac{1}{4}}(x-a) e^{-\frac{(x-a)^{2}}{2}} ; \\
\psi_{k}=(-1)^{k} \pi^{-\frac{1}{4}}\left(2^{k} k !\right){ }^{-\frac{1}{2}} e^{\frac{x^{2}}{2}} \frac{d^{k}}{d x^{k}} e^{-x^{2}}
\end{gathered}
$$

Интегралы (3б) с функциями (3в, Зг) являются хорошо известными в теории электронно-колебательных переходов интегралами перекрывания, ${ }^{\star \star}$ (см., напр., $\left.\left[{ }^{5}, 10,11\right]\right)$ :

$$
P_{0 k}=\left(2^{k} k !\right)^{-\frac{1}{2}} e^{-\frac{a^{2}}{4}} ; \quad P_{k 1}=\left(2^{k-1} k !\right)^{-\frac{1}{2}}\left(k-\frac{a^{2}}{2}\right) a^{k-1} e^{-\frac{a^{2}}{4}} \text {. }
$$

Подстановка (4) в (2) дает после небольших преобразований ответ в виде бесконечного ряда:

$$
S_{01}(\mu, a)=-\frac{2 \sqrt{2} e^{-\frac{a^{2}}{2}}}{a} \sum_{k=1}^{\infty} \frac{a^{2^{k}}}{2^{k}(k-1) !(2 k-1-\mu)(2 k+1-\mu)} .
$$

Таким образом, обычная форма теории возмущений дала представление $S_{01}(\mu, a)$ в виде разложения по степеням $a$, очень удобное при малых $a . S_{01}(\mu, a)$ стремится к нулю при $a \rightarrow 0$. Случай $\quad \mu \rightarrow 2 n+1$, где $n=0,1,2, \ldots$, соответствует рассеянию в области сильного поглощения и требует для своего рассмотрения учета конечной ширины уровня.

Единственным параметром молекулы, определяющим в рассматриваемом приближении интенсивность комбинационного рассеяния, является безразмерный сдвиг положения равновесия $a$. Значению $a=1$ соответствует, в размерных единицах сдвиг $\xi_{0}=\left(h / M \omega_{0}\right)^{\frac{1}{2}}$, где $M$ и $\omega_{0}$

* В теории контуров электронно-колебательных полос поглощения эти поправки учтены $[8,9]$.

** Их удобно вычислить, например, интегрнрованием по частям. 
масса и частота осциллятора. Величину $\xi_{0}$ можно представить себе как классическую амплитуду нулевого колебания осциллятора.

Часто приходится иметь дело со сдвигами $a>1$ (например $a \approx 5$ ). Особенно актуален случай $a>1$ в теории примесного центра в кристалле при рассмотрении локальных колебаний, наиболее сильно взаимодействующих с электронным переходом. Поэтому необходимо искать другие представления $S(\mu, a)$.

\section{4. Вспомогательное неоднородное дифференциальное уравнение}

Пусть требуется вычислить сумму вида

$$
S(\mu)=\sum_{k} \frac{\int f \psi_{k} d x \int g \psi_{k} d x}{\varepsilon_{k}-\mu}
$$

где функции $\psi_{k}$ образуют полный набор собственных функций некоторого самосопряженного оператора $\widehat{H}$ :

$$
\left(\widehat{H}-\varepsilon_{k}\right) \psi_{k}=0
$$

$\mu$ - параметр, а $f$ и $g$ - заданные функции.

Обозначим через Ф сумму

$$
\Phi=\sum_{k} \frac{\int f \psi_{k} d x}{\varepsilon_{k}-\mu} \psi_{k} .
$$

Легко увидеть, что Ф удовлетворяет дифференциальному уравнению

$$
(\widehat{H}-\mu) \Phi=f
$$

Для этого разложим решение уравнения (9) в ряд по функциям $\psi_{k}$

$$
\Phi=\sum_{k} c_{k} \psi_{k}
$$

подставим это разложение в (9), умножим на $\psi_{l}$ и проинтегрируем по $x$. В результате получаем:

$$
c_{l}=\frac{\int \psi_{l} f d x}{\varepsilon_{l}-\mu} .
$$

Формулы (10) и (11) показывают, что функция Ф (8) действительнс является решением уравнения (9). Сумма (6) записывается теперь, в виде

$$
S(\mu)=\int g \Phi d x
$$

Таким образом, задача расчета суммы $S(\mu)$ сводится к решению неоднородного дифференциального уравнения (9) при надлежащих предельных условиях и к квадратуре (12). При этом для нахождения суммы (6) в качестве функций $f$ и $g$ в формулах (9) и (12) надо положить либо $f=\varphi_{i}$ и $g=\varphi_{f}$, либо, наоборот, $f=\varphi_{i}$ и $g=\varphi_{i}$. 


\section{5. Связь с функцией Грина}

Нетрудно увидеть связь проведенного в предыдущем параграфе рассмотрения с обычной функцией Грина $G$. Действительно, с помощью функции $G$ решение уравнения (9) можно записать в виде интеграла

$$
\Phi(x)=\int G\left(x, x^{\prime} ; \mu\right) f\left(x^{\prime}\right) d x^{\prime},
$$

где $G\left(x, x^{\prime} ; \mu\right)$ удовлетворяет уравнению

$$
(\widehat{H}-\mu) G\left(x, x^{\prime} ; \mu\right)=\delta\left(x-x^{\prime}\right) .
$$

К связи с функцией Грина можно прийти и непосредственно из формулы (6). Для этого следует переставить местами суммирование и интегрирование и вспомнить, что функцию Грина можно представить в виде (см., напр., $\left[{ }^{12}\right]$ стр. 526)

$$
G\left(x, x^{\prime} ; \mu\right)=\sum_{k} \frac{\psi_{k}(x) \psi_{k}\left(x^{\prime}\right)}{\varepsilon_{k}-\mu} .
$$

В случае гамильтониана гармоннческого осциллятора функция Грина $G\left(x, x^{\prime} ; \mu\right)$ записывается так

$$
\begin{array}{ll}
G\left(x, x^{\prime} ; \mu\right)=\frac{1}{W(0)} g_{\mu}(x) g_{\mu}\left(-x^{\prime}\right) & \text { при } x \geqslant x^{\prime}, \\
G\left(x, x^{\prime} ; \mu\right)=\frac{1}{W(0)} g_{\mu}(-x) g_{\mu}\left(x^{\prime}\right) & \text { при } x \leqslant x^{\prime},
\end{array}
$$

где через $g_{\mu}(x)$ обозначено решение уравнения

$$
g_{\mu}^{\prime \prime}(x)+\left(\mu-x^{2}\right) g_{\mu}(x)=0,
$$

обращающееся в нуль при $x \rightarrow+\infty$, а

$$
W(0)=2 g_{\mu}^{\prime}(0) g_{\mu}(0)=g_{\mu}^{\prime}(x) g_{\mu}(-x)-g_{\mu}(x) g_{\mu}^{\prime}(-x)
$$

есть определитель Вронского, составленный из решений уравнения (14) $g_{\mu}(x)$ и $g_{\mu}(-x)$, которые являются линейно независимыми (в предположении, что $\mu$ не есть собственное значение, т. е. $\boldsymbol{\mu} \neq 1,3,5, \ldots$ ). Легко увидеть, что (с точностью до произвольного постоянного множителя)

$$
g_{\mu}(x)=D_{\frac{\mu-1}{2}}(x \overline{\sqrt{2}}),
$$

где $D_{p}(z)$ есть функция Вебера (функция параболического цилиндра), определенная так, что $D_{p}(z) \rightarrow 0$ при $z \rightarrow+\infty$.

\section{6. Простое решение в одном частном случае}

Запишем уравнение (9) для вероятности перехода гармонического осциллятора в результате комбинационного рассеяния света с нулевого $(i=0)$ на первый колебательный уровень $(f=1)$, полагая $f(x)=\varphi_{1}(x)$ :

$$
\Phi^{\prime \prime}+\left(\mu-x^{2}\right) \Phi=-\sqrt{2} \pi^{-\frac{1}{4}}(x-a) e^{-\frac{(x-a)^{2}}{2}}
$$

и рассмотрим частный случай $\mu=a^{2}+1$. При этом значении $\mu$ (16) имеет решение

$$
\Phi=\frac{\pi^{-\frac{1}{4}}}{a \sqrt{2}} e^{-\frac{(x-a)^{2}}{2}} .
$$


В этом случае мы имеем согласно (12) и (3б) после интегрирования

$$
S_{01}\left(a^{2}+1 ; a\right)=\frac{1}{a \sqrt{2}} .
$$

Полученный результат полезен для проверки общих формул для $S_{01}(\mu, a)$, а также в качестве «опорной точки» в численных расчетах.

Сопоставим полученный результат с обычной формой теории возмущений. Нетрудно увидеть, что (5) при $\mu=a^{2}+1$ сводится к (18), если справедливо соотношение

$$
\text { , } e^{y}=-\sum_{k=1}^{\infty} \frac{y^{k}}{(k-1) !(k-1-\mu)(k-y)} .
$$

Формула (19) является несколько необычным представлением экспоненциальной функцин. Справедливость этого представления в области $|y|<1$ доказывается путем небольшого преобразования суммы в правой части (19), а его справедливость для всех $y$ вытекает из принципа аналитического продолжения.

\section{7. Решение вспомогательного дифференциального уравнения методом контурного интегрирования}

Рассмотрим опять основной переход $(i=0 ; f=1)$. Возьмем уравнение (9) в следующем виде (полагая $\left.f(x)=\varphi_{0}(x)\right)-$

$$
\Phi^{\prime \prime}+\left(\mu-x^{2}\right) \Phi=-\pi^{-\frac{1}{4}} e^{-\frac{(x-a)^{2}}{2}} .
$$

После подстановки

$$
\Phi(x)=-\pi^{-\frac{1}{4}} e^{-\frac{x^{2}+a^{2}}{2}} u(x)
$$

приходим к уравнению

$$
u^{\prime \prime}-2 x u^{\prime}+(\mu-1) u=e^{a x} .
$$

Полученное неоднородное уравнение будем решать методом контурного интегрирования, применяемым обычно к однородным уравнениям. Решение будем искать в виде

$$
u(x)=\int_{l} e^{x t} v(t) d t
$$

Подстановка (23) в (22) дает:

$$
\int_{l} e^{x t}\left[2 t v^{\prime}+\left(t^{2}+\mu+1\right) v\right] d t-\left.2 t v e^{x t}\right|_{l}=e^{a x} .
$$

Для удовлетворения уравнению (24) достаточно потребовать, чтобы контурный интеграл равнялся нулі, а второй член слева и член справа взаимно сократились.

Первое условие приведет нас к дифференциальному уравнению для $v(t)$. Решение этого уравнения дает:

$$
v(t)=C e^{-\frac{t^{2}}{4}} t^{-\frac{\mu+1}{2}}
$$

Во втором условии заключено отличие применяемого нами метода от случая решения однородного уравнения, когда контур интегрирова ния $l$ следует выбрать так, чтобы второй член слева в (24) обратился в нуль. Мы обязаны найти такой контур, чтобы этот член стал равным $e^{a x}$. 
Свойства функции $v(t)$ и вместе с тем выб́ор контура интегрирования зависят от значения параметра $\mu$.

$$
\text { Сл у ч а й } \mu<\frac{1}{2}
$$

В качестве контура ннтегрирования $l$ можно выбрать отрезок вещественной оси от $t=0$ до $t=a$. Для постоянной интегрирования мы получаем

$$
C=-\frac{1}{2} e^{\frac{a^{2}}{4}} a^{\frac{\mu-1}{2}}
$$

Случай любых значений $\mu$, кроме $\mu= \pm 2 n+1$

(где $n$ целое)

За контур $l$ можно взять окружность радиуса $a$ вокруг начала координат. Для постоянной интегрирования мы получаем

$$
C=\frac{e^{\frac{a^{2}}{4}} a^{\frac{\mu-1}{2}}}{2\left(1+e^{-\mu \pi i}\right)}
$$

Для нахождения искомой суммы $S_{01}(\mu, a)$ следует выполнить еще интегрирование по $x$ согласно формуле $(12)$, в которой в качестве $g$ взята $\varphi f$ по (Зв). Мы получаем:

$$
\begin{gathered}
S_{01}(\mu, a)=C 2^{-\frac{1}{2}} e^{-\frac{3 a^{2}}{4}} \oint_{l} d t t^{-\frac{\mu+1}{2}}(a-t) e^{\frac{a t}{2}} . \\
\text { С л у ч а й } \mu<\frac{1}{2} \\
S_{01}(\mu, a)=2^{-\frac{3}{2}} e^{-\frac{a^{2}}{2}} a \int_{0}^{1} d \tau e^{\frac{a^{2}}{2} \tau} \tau \tau^{-\frac{\mu+1}{2}}(\tau-1) .
\end{gathered}
$$

Случай любых значений $\mu$, кроме $\mu= \pm 2 n+1$

$$
(n-\text { целое число) }
$$

$$
S_{01}(\mu, a)=-\frac{e^{-\frac{a^{2}}{2}} a}{2^{\frac{3}{2}}\left(e^{-\mu \pi i}+1\right)} \oint d \tau e^{\frac{a^{2}}{2} \tau} \tau^{-\frac{\mu+1}{2}}(\tau-1)
$$

где интегрирование проводится по единичной окружности с центром в начале координат.

В частном случае $\mu=-2 m+1$, где $m=1,2, \ldots$, интеграл (29) вычисляется в конечном виде и мы получаем

$$
\begin{gathered}
S_{01}(-2 m+1, a)=\frac{m ! \sqrt{\alpha}}{2}\left(\sum_{k=1}^{m+1} \frac{(-1)^{k} k}{(m+1-k) ! a^{k+1}}+\right. \\
\left.+\frac{(-1) m m !(\alpha+m+1)}{\alpha^{m+2}} e^{-\alpha}\right)
\end{gathered}
$$

где введено обозначение $\alpha=\frac{a^{2}}{2}$. 
Итак, проведенное рассмотрение привело нас к суммам $S_{01}(\mu, a)$, выраженным в виде интегралов. В принципе формула (29) уже пригодна для сравнительно простого численного интегрирования и составления таблиц $S_{01}(\mu, a)$ в актуальном диапазоне $\mu$ и $a$. Однако численный расчет в случае $\mu>\frac{1}{2}$ все же не очень удобен, так как контур интегрирования в (30) должен обходить начало координат, в котором подинтегральная функция имеет неинтегрируемую особенность. Для «стандартизации» способа расчета функции двух переменных $S_{01}(\mu, a)$ удобно иметь для нее несложное дифференциальное уравнение, имеющее силу при в сех $\mu$ (за исключением случаев резонанса $\mu=2 m+1, m=0,1, \ldots$ ). Вывод такого уравнения дается ниже.

\section{8. Дифференциальное уравнение для определения $S_{01}(\mu, a)$}

Введем в формулы (29) и (30) обозначения $\alpha \equiv \frac{a^{2}}{2}, \nu \equiv-\frac{1}{2}(\mu+1)$, $S_{01}(\sqrt{2 \alpha},-2 v-1) \equiv S(\alpha, v)$ и продифференцируем $S(v, \alpha)$ по $\alpha$. С помощью интегрирования по частям нетрудно показать, что функции $S(v, \alpha)$, определяемые формулами (29) и (30), удовлетворяют одному и тому же обыкновенному дифференциальному уравнению первого порядка:

$$
\frac{d S}{d \alpha}+\left(1+\frac{v+\frac{3}{2}}{\alpha}-\frac{1}{\alpha+v+1}\right) S=-\frac{1}{2 \sqrt{\alpha}(\alpha+v+1)}
$$

Вводя новую функцию $R=-2 \sqrt{\alpha}(\alpha+v+1) S$, получаем для нее уравнение

$$
\frac{d R}{d \alpha}+\left(1+\frac{v+1}{\alpha}-\frac{2}{\alpha+v+1}\right) R=1 .
$$

Уравнения (31) или (32) вместе с условием $S(v, 0)=0$ (см. (5)) определяют $S(v, \alpha)$ для всех $\alpha$ и $v$ и дают удобный способ численного нахождения $S(v, \alpha)$ во всем интервале значений $\alpha(0 \leqslant \alpha<\infty)$ с помощью электронно-счетных машин. Затруднения могли бы возникнуть при $\dot{v}<-1$ в окрестности точки $\alpha=-v-1$, но, согласно результатам $\S 6$, в этой точке известно точное значение

$$
S(v, \alpha): S(v,-v-1)=\frac{1}{2 \sqrt{-v-1}} .
$$

\section{9. Метод моментов и правила сумм}

Метод моментов оказался эффективным при вычислении вероятностей квантовых переходов, рассматриваемых в первом порядке теории возмущений. Этот метод, по существу, означает описанне распределения вероятностей переходов с помощью правил сумм, аналогичных известной теореме о сумме сил осцилляторов. В некоторых задачах, например, при расчете контуров сплошных электронно-колебательных полос примесей в кристаллах, такое описание дает практически полное решение (см., напр., $[8,9,13])$.

Методом моментов легко получить правила сумм и для произведения матричных элементов $P_{k j} P_{i k}$ в (2). Хотя получаемые выражения непосредственно не описывают нероятностей переходов, они могут оказаться полезными в качестве вспомогательных точных соотношений, которым должны удовлетворять бесконечные ряды по $k$ из про- 
изведений $P_{k f} P_{i k}$. Эти соотношения могут служнть критерием точности приближений, сделанных при вычислении матричных элементов. Это весьма важно, так как структура рядов теории возмущений такова, что убывание абсолютной величины членов ряда при больших $k$ только за счет роста знаменателя происходит медленно.

Будем рассматривать выражение

$$
S_{l}(\mu)=\sum_{k}\left(\varepsilon_{k}-\mu\right) l P_{k f} P_{i k}
$$

получающееся из формулы (2) при умножении $k$-го члена в ее правой части на $\left(\varepsilon_{k}-\mu\right)^{l+1}$. Оно является моментом (положительного) порядка $l$ «функции распределения» $P_{k f} P_{i k}{ }^{*}$. Суммирование по $k$ легко выполнимо, и мы получаем $\left[{ }^{14}\right]$ :

$$
S_{l}(\mu)=\sum_{p=0}^{l}(-1)^{l}\left(\begin{array}{l}
l \\
p
\end{array}\right) \mu^{p} \int d x\left[\widehat{P}+\varphi_{i}^{*} \widehat{H}_{\mathrm{II}}^{l-p} \widehat{P}_{\varphi_{f}} .\right.
$$

Считая оператор возмущения $\widehat{P}$ самосопряженным, мы имеем для первых моментов:

$$
\begin{aligned}
& S_{0}(\mu)=\int \varphi_{i}^{*} \widehat{P}^{2} \varphi_{f} d x, \\
& \left.S_{1}(\mu)=\int \varphi_{i}^{*} \widehat{\left(P H_{\mathrm{II}}\right.} \widehat{P}-\mu \widehat{P}^{2}\right) \varphi_{f} d x, \\
& \left.\dot{S}_{2}(\mu)=\int \varphi_{i}^{*} \widehat{\left(P H_{\mathrm{II}}^{2}\right.} \widehat{P}-2 \mu \widehat{P} \widehat{H}_{\mathrm{II}} P+\mu^{2} \widehat{P}^{2}\right) \varphi_{f} d x
\end{aligned}
$$

Шронллюстрируем соотношения (35) на примере задачи комбинационного рассеяння, рассмотренной в настоящей работе (адиабатическое приближение; приближение Кондона). Из (35) мы получаем:

$$
\begin{aligned}
S_{0}(\mu) & =\delta_{i f} \\
S_{1}(\mu) & =\int \varphi_{i}^{*} U(x) \varphi_{f} d x+\left(\varepsilon_{f}-\mu\right) \delta_{i j} . \\
S_{2}(\mu) & =\int \varphi_{i}{ }^{*}\left[U^{2}(x)+\widehat{H}_{\mathrm{I}} U(x)+U(x) \widehat{H}_{\mathrm{I}}\right] \varphi_{f} d x- \\
& -2 \mu \int \varphi_{i}^{*} U(x) \varphi_{f} d x+\left(\varepsilon_{f}-\mu\right)^{2} \delta_{i f}
\end{aligned}
$$

Здесь мы учли, что гамильтониан промежуточных состояний $\widehat{H}_{\mathrm{II}}=\widehat{H}_{\mathrm{I}}+U(x)$, где $\widehat{H}_{\mathrm{I}} \varphi_{f}=\varepsilon_{f} \varphi_{f}$, а $U(x)=\widehat{H}_{\mathrm{II}}-\widehat{H}_{\mathrm{I}}$ представляет собой разность между адиабатическими потенциалами рассматриваемых электрснных состояний. Пока мы не использовали предположения о гармоническом характере колебаний. Если ограничиться гармоническим приближением и не учитывать изменения частоты колебания при электронном переходе, то интегралы (36) легко вычисляются. Тогда следует положить $U(x)=$ $=b x+c$, а за $\varphi_{i}$ и $\varphi_{f}$ принять соответствующие волновые функции гармоннческого осциллятора (принадлежащие уже обе колебательному гамильтониану основного электронного состояния). Результат для $i=0, f=1$ будет иметь вид:

* Формулу (2) можно рассматривать как момент первого отрицательного порядка $S_{-1}(\mu)$ распределения (не являющегося положительно определенным) $P_{k f} P_{i k}$. Нетрудно увидеть, что все последующие моменты отрицательного порядка получаются из $S_{-1}(\mu)$ дифференцированием по параметру $\mu$. Из $\S 5$ следует связь $S_{-1}(\mu)$ с функщией Грина. 


$$
\begin{aligned}
S_{0}= & 0 \\
S_{1}= & b\left(\frac{h}{2 M \omega_{0}}\right)^{\frac{1}{2}} \\
S_{2}= & b\left(\frac{h}{2 M \omega_{0}}\right)^{\frac{1}{2}} 2\left(c-\mu+\frac{1}{4} h \omega_{0}\right), \\
S_{3}= & b\left(\frac{h}{2 M \omega_{0}}\right)^{\frac{1}{2}}\left[3 c^{2}+3 b^{2}\left(\frac{h}{2 M \omega_{0}}\right)+\frac{3}{2} c h \omega_{0}-\right. \\
& \left.-3 \mu\left(2 c-\mu+\frac{h \omega_{0}}{2}\right)+\frac{9}{16}\left(h \omega_{0}\right)^{2}\right] .
\end{aligned}
$$

Интегралы (36) очень легко вычисляются и в случае изменения колебательной частоты при электронном переходе, когда $U(x)=d x^{2}+b x+c$. Нетрудно вычислить также моменты $S_{l}$ более высоких порядков, однако нх выражения становятся довольно громоздкими.

Формула (34) является весьма общей: для ее вывода использовались лишь общие положения квантовой механики (полнота системы собственных функций, эрмитовость оператора энергии). В частности, применимость ее не ограничивается одномерной задачей. Можно надеяться, что в некоторых случаях получаемые из (34) моменты положительных порядков $S_{l}$ позволят с достаточной степенью точности определить и $S_{-1}$, т. е. амплитуду вероятности перехода по формуле (2). Действительно, в сумме (2) у членов, для которых $\left|\varepsilon_{k}\right|<|\mu|$, знаменатель можно разложить по положительным степеням отношения $\varepsilon_{k} / \mu$. Отдельные члены этого разложения будут выражаться через моменты положительных порядков, которые относительно легко вычисляются. Можно думать, что в некоторых задачах этот метод обеспечит достаточную точность уже при учете нескольких первых моментов положительного порядка. Более точное исследование этого вопроса целесообразно проводить для конкретных задач.

Соотношения, аналогичные (34), легко получить также для произведений матричных элементов, характерных для второго порядка стационарной теории возмущений. Небольшие и очевидные модификации способа определения моментов позволяют получить такого же типа соотношения для матричных элементов высших порядков теории возмущений.

\section{0. Заключительные замечания}

Рассмотренная задача комбинационного рассеяния относилась is модели двухатомной молекулы. Среди возможных расширений разобранной модели самой актуальной и интересной является задача комбинационного рассеяния света кристаллом с примесью, т. е. системой, где одновременно имеются кристаллические и локальные колебания. Можно думать, что последовательное рассмотрение этой задачи, аналогичное недавнему теоретическому рассмотрению теории квазилинейчатых электронно-колебательных спектров [15] (в частности, эффекта Шпольского [16]), приведет к теории квазилинейчатых спектров комбинационного рассеяния.

Авторы признательны Л. Л. Крушинскому и П. П. Шорыгину, беседы с которыми привлекли внимание к рассмотренному кругу вопросов, и Р. А. Преэму за многочисленные обсуждения.

\section{Л ИТЕ Р А Т У Р А}

1. Э. В. Ш польски й, УФН, $68,51,1960$.

2. П. П. Шорыгин, ЖФХ, 25, 241, 1951; П. П. Шорыгин, Л. Л. Крушин с к и й, Материалы Х Совещ. по спектроскопии, 1, 215, 1957.

3. Л. Л. К р ушин ский, П. П. Шор ыгин, Опт. и спектр., 11, 24, 151, 1961.

4. Л. Л. К р ушн н с к и й, Диссертация, Минск, 1962. 
5. Р. А. Преэм, Тр. ИФА АН ЭССР, № $16,57,1961$.

6. Р. А. П реэм, Тр. ИФА АН ЭССР (в печати).

7. Д. И. Блохи нц ев, Основы квантовой механики, 1961.

8. К. К. Ребане, А. П. Пурга, О. И. Сильд, В. В. Хи жняков, Тр. ИФА АН ЭССР, № 14, 31, 48, 1961.

9. О. И. С нльд. Тр. ИФА АН ЭССР, № 15, 21, 1961.

10. К. К. Ребане, А. А. Рентель, О. И. Сильд. Инженерно-физ. журнал, 2. $60,1959$.

11. M. W a g ne r, Zs. Naturforsch., 14a, 81, 1959.

12. В. И. Сми н нов, Курс высшей математикн, т. IV, 1957.

13. Н. Н. К ри сто фель, К. К. Реб ане, Физика щелочно-галоидных кристаллов, стр. 32. Рига, 1962.

14. А. П. Пурга, К. К. Реб ане, Тр. ИФА АН ЭССР, 19, 146, 1962.

15. К. К. Р ебане, В. В. Хи жняков, Тезисы дскладов на ХI Совещании по люминесценции, Минск, 1962; Оптика и спектроскопия, 14, 362, 491, 1963.

16. Э. В. Ш польски й, УФН, $71,215,1960 ; 77,321,1962$.
Институт физики и астрономии
Академии наук Эстонской ССР
Поступила в редакцию
Ленинградский государственный университет 16. XII 1962

\title{
VALGUSE KOMBINATSIOONHAJUMISE TEOORIAST MOLEKULIL
}

\author{
K. Rebane, \\ Eesti NSV Teaduste Akadeemia korrespondeeriv liige
}

T. Rebane,

füüsika-matemaatikateaduste kandidaat

Resümee

Vaadeldakse kombinatsioonhajumise intensiivsust molekuli vōnkumisel adiabaatse lähendusmeetodi alusel. Vōnkumist kirjeldatakse harmoonilise ostsillaatori abil, mille tasakaalupunkt nihkub molekuli elektronoleku muutumisel, võnkesagedus aga jääb endiseks. Vastava kvantmehhaanilise teist järku häiritusteooria ülesande lahendamiseks on välja töötatud uus meetod, mille abil summeeriti üle vaheseisundite. Käsitletakse mõningaid üldisi probleeme, mis on seotud teist järku häiritusteooria ülesannetega: uuritakse nende ülesannete seost Greeni funktsiooniga, saadakse harmoonilise ostsillaatori statsionaarse Schrödingeri vôrrandi Greeni funktsioon, tuletatakse summareeglid teist järku häiritusteooria maatrikselementide jaoks.
Eesti NSV Teaduste Akadeemia
Füüsika ja Astronoomia. Instituut
Saabus toimetusse
16. XII 1962

\author{
Leningradi Riiklik Olikool
}

\section{ON THE THEORY OF THE RAMAN EFFECT ON MOLECULES}

\author{
K. Rebane, \\ Corresponding Member of the Academy of Sciences of the Estonian S.S.R. \\ T. Rebane \\ Summary.
}

The intensity of the Raman scattering by molecular oscillations is calculated on the basis of the adiabatic approximation. The oscillations are described in terms of harmonic oscillators whose equilibrium positions are displaced with the change of the electronic state of the molecule, but whose frequencies remain unchanged.

For the solution of the corresponding quantum mechanical second order perturbation theory problem a new method is applied, permitting to sum up all intermediary states. Also some general problems connected with the second order perturbation theory are discussed, as the relation of such problems with Green's functions, and Green's function for the harmonic oscillator's stationary Schrödinger equation is obtained. Lastly, some sum rules for the second order perturbation theory matrix elements are derived.

\footnotetext{
Academy of Sciences of the Estonian S.S.R., Institute of Physics and Astronomy

Leningrad State University
}

\section{Received}

Dec. 16th, 1962 